Editorial

\title{
Tides of Change in Administration Affect Management but not the Desire to Care
}

\section{Joanne Loewy ${ }^{1,2}$ Ralph Spintge ${ }^{3,4}$}

${ }^{1}$ The Louis Armstrong Center for Music \& Medicine, Mount Sinai Beth Israel, New York, NY, USA

${ }^{2}$ Icahn School of Medicine, New York, NY, USA

${ }^{3}$ Deptartment of Algesiology and Interdisciplinary Pain Medicine, Regional Pain Centre DGS, Sportklinik Hellersen, Lüdenscheid, Germany

${ }^{4}$ Institute for Music Therapy, University for Music and Drama HfMT Hamburg, Germany

A compelling post released some years ago on the Harvard Business Review called "First Let's Fire All the Managers" [1] prompts recent thinking about the developing trends in healthcare administration. Being in the work, as hospitalists, the opportunity to witness turnover is not unfamiliar. On some accounts there are political regime changes that have resulted in new leadership. On other occasions there are community pressures, such as the moving trend of care-flow to be less acute in hopes that people will be moved to become more adherent about taking care of themselves. The movement from in-patient, acute care status, toward a strong impetus to take a preventative stance, and one where people avoid hospitalization by taking better notice of their health when well, is still an apparent and growing orientation. Preventative medicine's focus on prevention-diet, exercise and the development of wellness practices, resulting from such shifts has expanded. As a consumer, the patient has the opportunity to make many choices. Web rating sites have perhaps led to healthcare professionals and institutions taking more accountability of their actions. It is not only the Joint Commission or outside accrediting or certifying agencies that keep standards of healthcare high. In current times, the consumers, our patients, can go online and write about how wonderful or horrific they have experienced their hospital stay or exactly how their healthcare professional treated them, nurturing or neglectful.

As Editors, we have numerous opportunities to visit hospitals and programs involving healthcare in other countries. It is not surprising that each institution, country, unit and staff have their own set of rules, and individualized

\section{PRODUCTION NOTES: Address correspondence to:}

Joanne V. Loewy DA, LCAT, MT-BC, Address: The Louis Armstrong Center for Music and Medicine, Mount Sinai Beth Israel, $16^{\text {th }}$ and $1^{\text {st }}$ Avenue, 6 Silver 21, New York, NY 10003, USA. Email: jloewy@chpnet.org | COI statement: The authors declared that no financial support was given for the writing of this article. The authors have no conflict of interest to declare. standards of care. It is always fascinating to evaluate the group process and dynamics of staff interaction where the care of people are concerned. In the aforementioned Harvard Review, Gary Hamel writes about the new trends of leadership and accountability. He has looked carefully at companies that are profitable and where employees are happy and notes a lack of concern about "empowerment" as he describes one:

\footnotetext{
During my visit, I didn't hear anyone use the term "empowerment." That's because the notion of empowerment assumes that authority trickles down-that power gets bestowed from above, as and when the powerful see fit. In an organization built on the principles of self-management, individuals aren't given power by the higher-ups; they simply have it [1 pg. 3-4].
}

Hamel discusses the growing value of working peer to peer without an autocratic system of top down. Peer groups, in such companies, make team decisions. There is less 'lower' and 'higher' and even areas such as compensation are transparent, and can generate as the result of what happens in group recommendations, meaning that the people that are involved are deciding-rather than on viewing the particular of 'who' is paid 'what.' The content and levels of what employees should be paid are the focus of everyone, not an exclusive group at the top. Transparency and accountability are set by working teams that manage the growth and set precedence according to elements, such as, for instance, the company's prudency. People compete for value, and colleague weigh-in to address the detailed problem solving. Salaries are compensated by the value added to the systems created by the working teams. The approach is seemingly non-authoritative. They train their first-level employees in content-financial analysis. This means that front-line workers make the decisions on things such as, what equipment is needed, and why it should be purchased. This translates to less time negotiating-and more action-driven hands-on work.

How much might it be to our advantage to consider less hierarchy with an increase of informed decisions made by people with their hands directly on patients? In this kind of environment, everyone has power-and the decision of how and who 'takes it on' is determined by the relational fit to the 
work need. Hamel has studied what he describes as these highly synchronized companies, such as 'Morning Star Company' and others. He points out that an ideology of management is centralism. Folks who are free to contract and where decision-making is highly distributed-fair better. Conforming to effective work management with ladders can undermine freedom and the process of engagement. People desire the freedom and flexibility of the market-the capacity to access executive control of coordinated and synchronized working relationships without the ladder. Starting from the ideology of engagement, he argues that control and coordination has its advantages without sacrificing creativity and decision-making.

Rules and procedures can undermine engagement and creativity and control can be maintained without sacrificing vision. Yet, he questions, how do we strategically give the freedom where people can harvest their visions without compensating trust, while keeping ample efficacy that adheres to rules and structure.

Interestingly, there has been a recent trend for music therapists and frontline doctors to retire earlier or, to go back. I have met up with colleagues who have achieved top-line administrative roles, such as Chair, or Director and whom have attained freedom of sorts. In a hierarchy, the amount of computer work and paperwork can strangle the flow of a workday. Going back means that these colleagues will 'step back.' (Purposefully the term, 'step down' is not used here). These colleagues are longing to return to the work of treating. Rather than meetings of observing, delegating supervising, ordering, or even writing about, the desire is to treat patients and families. One such colleague said to me:

"For years I have worked my way up....I am now at the top, and I feel as if I did what I need to do, for the Department and within the system-but, for what? I long for the days where I could see patients eye to eye, and remember their faces, and stories. Next year, I'm not retiring, I'm going back."

It may be critical to always keep our hands and hearts with patients. There seems to be an indirect but parallel theme here. We ask patients to maintain their health by keeping an eye on themselves and by being adherent to what keeps them well. In a sense we are asking them to be their own doctor. Of course, this is not in the completely literal sense-what is encouraged is, that there be a growing adherence to a patient's capacity to take ownership of their health, which in turn helps diagnostics, evaluation and provides for more informed treatment.

As the trends of our work, and the business of managing and leading will be distributed out to the periphery, with less cast systems, leaders will emerge naturally by virtue of service to the community. So too will the trends of improved health care of patients-because as out-patient care increases, people will be given the authority and trust of managing their own wellness practices.

In turn, this ripple, unfolding as the scope of employee freedom that Hamel writes about grows, we will reflect upon the notion that people are accountable to their peers, rather than their managers. So too, in turn, will patients build their trust of doctor-therapist informed care, as they learn to dignify and trust the management of their learning, and their experiences of disease treatment and wellness.

Stepping back into the front line role of doctor, nurse, therapist or coach takes courage because it takes a certain amount of risk to uphold that such ideals and values of treating someone are both emotionally fulfilling and at the same time are dignified. If one has to use, as Hamel says, "the big stick of authority to get something done, then such leadership is short-lived." If patients wait until a volcano erupts and they count on hospitalization to adhere to wellness and prevention, than a resulting cure will be short-lived.

Power comes from serving the needs of the community. Good care instills that the pressure of healing or work success comes from teams, and relies on individuals to succeed. This is the place where monetary pay should not necessarily exist as the result of a role-or title. Accountability and freedom keep people highly engaged in their work. In the same way, the knowledge we impart to patients, and their importance of monitoring self-care when well, will translate to better outcomes in treatment regimens. Change is infused by the power we can infiltrate with human trust. And the passion we embark upon in treating human beings with music and medicine is a relationship that remains unsurpassed.

This issue starts with a paper by Kathy J. Helzlsouer, Kelly Phair, Shannon Manocheh and Stephen A. Holmes elaborating on a Feasibility Study of a Music Therapy Intervention for Patients Breast Cancer Survivors with Cognitive Dysfunction: The MusIC Study. While chemotherapy in cancer provides more and more specific and effective treatment for various kinds of cancer, undesired side effects are a major concern. Cognitive impairment called Chemobrain is of course also a highly emotional issue for cancer patients. Thus, studies in the field have to be undertaken in a sensitive way, particularly as the treatment of Chemobrain effects still remains elusive, as the authors clearly state.

The role that cultural background plays in both subjects and investigators in music and medicine is the topic of Sue Baine's article, The Role of Culture in Music and Medicine: Considerations to Enhance Health, she discusses social, ethical and political implications for music therapists, and highlights aspects to be considered as central components of selfreflection in their work.

John F. Mondanaro, Mark Ettenberger, Laurie Park in Mars Rising: Music Therapy and the Increasing Presence of Fathers in the NICU illustrate through analysis of case vignettes, the significance of balancing the role of both father 
and mother in the NICU environment. Their sensitive perspective of both parents, particularly in aspects related to music-culture enhances our vision and knowledge of best practices, as part of an expanded family-centered care treatment philosophy.

Nandhu Radhakrishnan, S.R. Savithri, and Rammohan Gangisetty describe the communication of emotions through carnatic vocal music observing variations in laryngeal dynamics of joy and sorrow in Expression of Emotions in Carnatic Vocal Music. Targeting patients suffering for instance, from Parkinson's disease, their approach should promise to find new ways of improving care in this field of steadily growing importance in health care and society in general.

In Addressing Community Concerns Through Music Penny A. Brill, a member of the viola section of the Pittsburgh Symphony Orchestra, and cancer survivor describes how she developed andimplemented support for her Pittsburgh Symphony Music and Wellness program and how teaming with professional music therapists can enhance the impact of orchestral community-based programs significantly, opening new opportunities to address community concerns.

In their pilot study, The Effects of Music Therapy on the Prosocial Behaviors of Adults with Disabilities Haley Rochelle Baumgarten and Barbara L. Wheeler target to enhance social behavior in disabled adults to attend their daily rehab programs through introducing music therapy interventions. Social interaction is a field of growing interest in health care in general, as ever more self-reliable and self-caring concepts are requested as part of integrative approaches. So further research may be highly recommended on this issue. This research provides a formidable beginning.

Michelle Nelson, Bev Foster, Sarah Pearson, Aimee Berends, Jennifer Ridgway, Renee Lyons, and Lee Bartel provide a multi professional study in Optimizing Music in Complex Rehabilitation and Continuing Care which focuses on the feasibility of so-called music care on one of 10 domains they have identified as target areas. While developing a conceptual framework of music care, as a research tool, their intension is to standardize such music interventions. We expect further studies to validate this approach and its suitability for other facilities, and to refine method and concept as to enhance the impact of such programs distinctly defining particular types of music care delivery. Again, this might be a way to meet rising complexity in integrative health care systems of today and tomorrow.

Such exciting variety of innovative research into music and medicine in a steadily growing arena related to aspects of treatment is accompanied by a thorough review of a book about the neuroscience of music with its implications in brain function and therapy, authored by Jordi A. Jauset, a renowned communications engineer. Reviewer Oscar Bedford invites readers from neuroscience, psychology and musicology to enjoy discovering music's many properties in his review of Cerebro y Música, Una Pareja Saludable, by Jordi A. Jauset. And finally, Marjorie Lee Jacobs takes the reader on a poetic journey using Violins of Hope.

It is our hope that this collection of stimulating articles may again foster advancement of music in medicine in its many facets. It may as well, prompt you to submit your own research studies for publication. The Editors are looking forward to welcoming you as members of a steadily growing global community as multidisciplinary faculty who are devoted to the sdevelopment of music in health care.

\section{References}

1. Hamel, G (2011) First Let's Fire all the Managers. Harvard Business Review dec 2011; retrieved 23-07-2016 from: https://hbr.org/2011/12/first-lets-fire-all-the-managers 